\title{
La educación universitaria en derechos humanos: el recurso a la historia como instrumento didáctico en Ciencias Sociales y Jurídicas
}

Higher education in Human Rights: turning to history as a teaching instrument in Social and Legal Sciences

Emilio Lecuona Prats

Profesor Contratado Doctor. Historia del Derecho.

Universidad de Málaga

E-mail: elp@uma.es

\begin{abstract}
Resumen: La educación en derechos humanos es una realidad a veces más teórica que práctica. Pese a las grandes declaraciones internacionales a su favor, diversos motivos juegan en su contra, tanto de tipo político como pedagógico. La didáctica de su enseñanza sigue siendo, por eso, un reto en la Universidad española. El presente estudio reflexiona acerca de la misma en el ámbito de las ciencias sociales y jurídicas mediante un triple análisis. En primer lugar, se hace un repaso histórico de la educación en derechos humanos tomando como referente las Naciones Unidas, desde la Declaración de 1948 hasta los Objetivos de Desarrollo Sostenible. A continuación, el interés se desplaza a España con la intención de hacer balance de su desarrollo en la práctica, a través de varios
\end{abstract}


informes de Amnistía Internacional. Por último, se hacen algunas observaciones sobre cómo debe ser su enseñanza, y la importancia que la Historia, tanto de los derechos humanos como de la educación en ellos, tiene para comprenderlos en su justa medida.

Palabras clave: Educación en Derechos Humanos, Derecho a la educación. Didáctica de los Derechos Humanos, Historia de los Derechos Humanos, Educación para la Comprensión Internacional, Objetivos de Desarrollo del Milenio (ODM), Objetivos de Desarrollo Sostenible (ODS), Informes de Amnistía Internacional en materia de educación.

Abstract: Human rights education is a reality sometimes more theoretical than practical. Despite the important international declarations in its favor, many reasons play against it, both political and pedagogical. The strategies for teaching human rights continues to be, therefore, a challenge in the Spanish University. This study reflects on this challenge both in the field of social and legal sciences through a triple analysis. First, a historical review of Human Rights Education taking the United Nations as a reference, from the 1948 Declaration to the Sustainable Development Goals. Next, the interest shifts to Spain with the intention of describing its development in practice, through various Amnesty International reports. Finally, some observations about how its teaching should be, and the importance of History, both of human rights and their teaching, in order their proper understanding.

Keywords: Human Rights Education, Right to education. Strategies for teaching Human Rights, Human Rights History. Teaching towards International Understanding, Millennium Development Goals (MDGs), Sustainable Development Goals (SDGs), Amnesty International reports on teaching.

Sumario: 1. Introducción. 2. La educación en derechos humanos: de la Declaración de 1948 a los Objetivos del Desarrollo Sostenible (ODS). 2.1. El derecho a la educación y el origen de la educación en derechos humanos. 2.2. El decenio de las Naciones Unidas para la educación en derechos humanos. 2.3. La educación en derechos humanos desde 2004 hasta el presente. 3. La enseñanza en derechos humanos en la universidad española. 4. Didáctica de los derechos humanos y la perspectiva histórico-jurídica. 4.1. Los obstáculos para una educación en derechos humanos en el Sistema Educativo Español, 
en particular el universitario. 4.2. La didáctica de los derechos humanos en el aula: especial referencia a la historia como recurso educativo. 5. Bibliografía.

\section{Introducción}

Los Derechos humanos son la gran aportación jurídica de la Edad Contemporánea. Con una partida de nacimiento clara, vinculada al Liberalismo y las Revoluciones Liberales, supusieron, en su inicio, una forma radicalmente nueva de comprender al ser humano dentro del Estado, defendiendo la esencia de su individualidad frente a éste, hasta convertirse, a la larga, en la piedra angular de todo sistema democrático.

La aparente sencillez de su planteamiento (tan alejado del tecnicismo de otras construcciones jurídicas), su comprensibilidad desde diversas ópticas culturales y niveles educativos, las evidentes ventajas sociales que ofrece su reconocimiento, su carácter dinámico, flexible, adaptable a las nuevas necesidades humanas y, en definitiva, su capacidad de precipitación “en cascada” (HUNT, 2009, 150 y 219), han coadyuvado a su aceptación, hasta el punto de que, por un proceso de difusión cultural realmente llamativo en la historia jurídica, han pasado, en muy poco tiempo, de ser una manifestación cultural de raigambre occidental, a convertirse en parte del acervo cultural de toda la humanidad, positivizándose incluso internacionalmente a partir de 1948.

Son, sin embargo, estos mismos caracteres que han promovido su extraordinaria divulgación, los que, paradójicamente, pueden debilitarlos. Los derechos humanos pueden así morir de éxito cuando todo el mundo los acepta desde cualquier perspectiva política y cultural por haberse convertido en un lugar común, pero de invocación más formal que práctica; cuando se desconoce que sólo son posibles en un ámbito social de tolerancia, democracia y libertad; cuando se simplifican en exceso, convirtiéndose en realidades ahistóricas, doctrinales, como si siempre hubiesen estado ahí; cuando se olvida que no son solo una idea moral (que lo son), sino que son derecho aplicable; cuando se multiplican hasta la infinidad amparándose en una interpretación inadecuada de su desarrollo en cascada; o cuando se convierten en un parapeto, una barrera, que se instrumentaliza políticamente para excluir cualquier opinión discrepante, catalogando sin más como contraria a los derechos humanos simplemente por no coincidir con la nuestra. 
Los derechos humanos son, por tanto, un logro mucho más delicado de lo que pudiera pensarse inicialmente y una forma de mantenerlos, de luchar contra lo señalado con anterioridad, no sólo está en sostener un buen aparato institucional que los lleve a la práctica (políticas públicas, tribunales,...), sino que, en especial, como ha reiterado la comunidad internacional, en convertirlos en una pieza esencial del ideario colectivo mediante la educación, es decir, entenderlos como una pieza de empoderamiento ciudadano que, desde un conocimiento individual y social de los mismos, convierta a toda la sociedad en su principal garante.

La educación en derechos humanos corre sin embargo el mismo peligro de morir de éxito que los propios derechos, pues su evidente aceptación, tanto a nivel internacional como nacional, no siempre garantiza su efectividad en la práctica, pues, como se señalaba ya hace más de una década, la dificultad estriba en pasar del "discurso político a las prácticas educativas" (CARIDE GÓMEZ, 2007, 329), es decir, del decir al hacer.

Varios son los motivos que siguen haciendo aún complejo plasmar en la realidad una educación en derechos humanos: en primer lugar, la necesidad de que dicha actividad deba adaptarse a diversos niveles educativos formales e informales. En segundo, a la disparidad de políticas educativas existentes para su enfoque (tanto a escala mundial, como nacional) en un panorama de pluralismo político e ideológico polarizado. Y, en tercer lugar, porque el tema pedagógico, esto es, la forma concreta en que debe educarse en derechos humanos pasa por multitud de retos no siempre correctamente concretados, desde la adecuada inclusión en el currículo educativo de los mismos (educación transversal o especializada) a la necesaria formación del docente o educador que de algún modo ha de impartirlos, pasando por la didáctica que debe emplearse para ello.

A lo largo de este artículo voy a centrarme especialmente en este último aspecto, es decir, el de la didáctica en la enseñanza de los Derechos Humanos, si bien con un foco limitado a un concreto nivel educativo (la enseñanza universitaria), un país determinado (España), y unos estudios específicos (esencialmente los Grados y Posgrados en el ámbito de las Ciencias sociales y jurídicas). El motivo de esta concreción se justifica tanto en que son una realidad que conozco desde mi experiencia como docente que lleva años tratando de introducir en sus asignaturas contenidos en Derechos Humanos (en concreto en asignaturas de Historia del derecho, Historia de las Relaciones Laborales e Historia de las Administraciones Públicas), como en el convencimiento de que en el ámbito de las 
ciencias sociales y jurídicas la educación en derechos humanos no debe sólo impregnar su currículo (de forma transversal) sino que deben enseñarse también de forma concreta, mediante asignaturas específicas de derechos humanos. Al hacerlo, insistiré además en cómo la Historia debe ser un recurso esencial didáctico para hacerlo, pues un correcto entendimiento de los derechos humanos pasa por comprenderlos como lo que son: productos culturales y, por tanto, historiables. Para ello, comenzaré haciendo un breve repaso histórico de la propia educación en derechos humanos en el ámbito de las Naciones Unidas, pues a pesar de existir valiosos estudios recientes sobre el tema, incluso con referencia al Consejo de Europa (BELLVER CAPELLA, 2017) me ha parecido esencial volver a narrarlo, insistiendo en sus aspectos didácticos.

\section{La educación en derechos humanos: de la Declaración de 1948 a los Objetivos del} Desarrollo Sostenible (ODS)

La necesidad de que los derechos humanos sean enseñados, es decir, sean mostrados, conocidos por la ciudadanía, ha sido probablemente una idea tan antigua como la publicación de estos a través de las primeras Declaraciones y un objetivo luego continuado por otros textos de diversa índole jurídica o extrajurídica, como por ejemplo los llamados Catecismos Políticos del siglo XIX. De este modo, podría afirmarse que la enseñanza de los derechos humanos fue no sólo anterior a la propia configuración del derecho a la educación, sino que, por supuesto, a una educación en los mismos.

\subsection{El derecho a la educación y el origen de la educación en derechos humanos}

El derecho a la educación no surge, como es sabido, en los momentos iniciales del liberalismo clásico. Por el contrario, en los primeros textos liberales, lo que prevalecen son las libertades individuales (negativas y positivas) frente al Estado, pero no actuaciones directamente exigibles a éste. Por este motivo, la libertad de enseñanza, e incluso la libertad de aprender, fueron también previas a un derecho a la educación en sentido estricto. Ciertamente, en algunos de los primeros textos constitucionales liberales (como las Constituciones francesas de 1791 y 1793, o la española de 1812) aparece ya recogido el compromiso político a una Instrucción Pública, pero es evidente que la consideración de la educación como derecho sólo se fue perfilando a lo largo del siglo XIX, conforme el Estado liberal fue superando su inicial abstencionismo social. Fue a 
partir de entonces, cuando el derecho a la educación surgió claramente de forma más o menos sincrónica en los diversos países y cuando, mientras lo hacía, fue manifestándose otro tema: el del "Estado Docente", es decir, el de la autoridad política que regula "la escolarización masiva de la población y su organización sistémica secular” (RUIZ, 2020, $551)$.

El origen de una educación en derechos humanos está muy conectada con este hecho, pues asumida por el Estado dicha labor educativa, surgió de inmediato el tema de los valores que tendrían que transmitirse mediante ella, pues por mucho que algunos liberales pudieran encontrar contradictorio con su papel garante de la libertad de enseñanza la transmisión de unos valores determinados, esto no sólo resultaba inevitable, sino que necesario para mantener el propio sistema liberal frente a otras ideologías contrarias de tipo autoritario como el comunismo o el fascismo.

No es extraño, por eso, que el derecho a la educación y, en concreto, la educación en derechos humanos, se plasme de forma clara tras el duro aprendizaje histórico que supuso la II Guerra Mundial y los crímenes cometidos durante ella, ni tampoco que fuera la ONU, fundada en otoño de 1945, la gran defensora de su implementación. Así, en la "Declaración Universal” de 1948 se consagra tanto un derecho a la educación a nivel mundial (art. 26.1), como una educación que "tendrá por objeto el pleno desarrollo de la personalidad humana y el fortalecimiento del respeto a los derechos humanos y a las libertades fundamentales" (art. 26.2).

Habrá que esperar sin embargo unos años para que este interés por una educación enfocada en los derechos humanos se plasme de forma más concreta en el ámbito internacional. Fue en la Conferencia Internacional de Teherán de 1968 cuando una de sus resoluciones, la XX, se centre precisamente en "la educación de la juventud en el respeto de los derechos humanos y en las libertades fundamentales", pidiendo "a los Estados (...) que velen por que se empleen todos los medios de educación para que los jóvenes crezcan y se desarrollen en un espíritu de respeto por la dignidad humana y por la igualdad de derechos de todos los hombres y todos los pueblos" (Final Act of the International Conference on Human Rights, 1968, 15-16). Exhortación que hizo extensiva a los organismos internacionales, en especial a la ONU y a la UNESCO. 
Más importante fue en cualquier caso una Recomendación adoptada por la Conferencia General de la Unesco el 19 de noviembre de 1974 sobre la Educación para la Comprensión, la Cooperación y la Paz Internacionales y la Educación relativa a los Derechos Humanos y las Libertades Fundamentales, en la que recomendaba adoptar lo que se catalogaba de "educación para la comprensión internacional". En primer lugar, porque se pedía a "cada Estado Miembro" que formulase y aplicase libremente (de acuerdo con su "práctica constitucional”) "una política nacional encaminada a aumentar la eficacia de la educación en todas sus formas" y en particular de los derechos humanos en todos los niveles educativos. En segundo lugar, porque insistía en el papel prioritario que en dicha educación debían tener los propios protagonistas del proceso educativo (docente y estudiantes). Y, en tercero, porque aportaba algunas indicaciones didácticas: “Los Estados Miembros deberían incitar a los educadores a poner en práctica (...) métodos que, apelando a la imaginación creadora de los niños y de los adolescentes (...) preparen a éstos a ejercer sus derechos y gozar de sus libertades, sin dejar de reconocer y respetar los derechos de los demás, y a cumplir sus funciones en la sociedad" (Recomendación sobre la Educación para la Comprensión, la Cooperación y la Paz Internacionales, 1974, 155).

El éxito de la Recomendación fue limitado. Aunque tuvo el mérito de plantear por vez primera de forma sistemática y práctica la enseñanza de los derechos humanos (aunque bajo la denominación de educación para la comprensión internacional), tres años más tarde, en 1977, la Comisión de Derechos Humanos de la ONU, volvía a recordarla al entender que la celebración del treinta aniversario de la Declaración de 1948, que iba a tener lugar el año siguiente podría suponer una nueva oportunidad para cumplir efectivamente con su objetivo, tanto por los Estados Miembros como, en especial, por "las escuelas y universidades, profesores y los padres de estudiantes" (Resolución 3/1977 de la Comisión de Derechos Humanos, 72-73).

En 1978 no se vivieron, pese a ello, grandes avances en esta materia. Sin embargo, un Congreso Internacional sobre la Enseñanza de los Derechos Humanos organizado por la UNESCO en septiembre de ese año, volvió a insistir en la relación existente entre educación y derechos humanos, añadiendo que ésta debía ser para todos y a lo largo de toda la vida recomendó que se celebrase una Convención y un "Plan Sexenal" sobre el tema; algo reiterado también por su Consejo Ejecutivo, que instó a que se profundizara 
en ello, con "un enfoque intersectorial multidisciplinario" (Decisiones aprobadas por el Consejo Ejecutivo de la UNESCO en su 105ª reunión, 1978, 44).

La enseñanza de los derechos humanos, pese a todo, siguió teniendo un desarrollo muy inferior al esperable, y habrá que esperar a que pasen casi quince años para que realmente comience a verse como una auténtica prioridad internacional, pues a pesar de alguna mención aislada (la Conferencia Mundial de Jomtién de 1990 sobre "Educación para todos"), no fue hasta 1993 que vieron la luz cuatro textos esenciales para su desarrollo efectivo: el "Plan Mundial de Acción para la educación en pro de los derechos humanos y la democracia" del "Congreso Internacional sobre la Enseñanza de los Derechos Humanos y la Democracia" convocado por la UNESCO en Montreal; la Resolución 1993/56 de la Comisión de Derechos Humanos, sobre "Educación y derechos humanos"; los párrafos 78 a 82 relativos a la "educación en Derechos Humanos" de la "Declaración y Programa de Acción de Viena" adoptada por la Conferencia Mundial de Derechos Humanos celebrada en dicha ciudad; y la resolución de la Asamblea General de las Naciones Unidas (48/127) que anunció el Decenio de las Naciones Unidas para la educación en la esfera de los derechos humanos.

Respecto al primero, se trata de un plan que pide "una movilización global de energías y recursos, desde la familia hasta las Naciones Unidas, para educar a las personas y grupos sobre los derechos humanos, de modo que se modifique la conducta que conduzca a la negación de los derechos, se respeten todos los derechos y se respeten los derechos civiles", aclarando que "el aprendizaje no es un fin en sí mismo, sino un medio para eliminar las violaciones de los derechos humanos y construir una cultura de paz basada en la democracia, el desarrollo, la tolerancia y el respeto mutuo" (Plan Mundial de Acción para la educación en pro de los derechos humanos y la democracia, 1993).

En cuanto a la Resolución de la Comisión de Derecho Humanos, en ella, se pedía convertir el conocimiento, tanto teórico como práctico, de los derechos humanos en un "cuestión prioritaria dentro de las políticas educativas", alabando el esfuerzo de los Estados que ya habían introducido en sus currículos esta materia, e invitando a hacerlo a los que aún no lo habían hecho para lograr una "educación integral", respetuosa con la diversidad étnica y los grupos más desprotegidos (indígenas, mujeres, niños, discapacitados...). Para ello pedía también la colaboración de los organismos 
internacionales y, en particular, de la Asamblea General de la ONU, con el fin de que promoviesen "programas de educación en derechos humanos y de promoción de la alfabetización" con fondos suficientes y para que se avanzara en la declaración de un decenio para la educación en derechos humanos (Resolución 56/1993 de la Comisión de Derechos Humanos, 193-195).

Las acciones 78 a 82 relativas a la "educación en Derechos Humanos" de la "Declaración y Programa de Acción de Viena" también insisten en todas estas ideas, reiterando que "los gobiernos, con la asistencia de organizaciones intergubernamentales, instituciones nacionales y organizaciones no gubernamentales, deben fomentar una mayor comprensión de los derechos humanos y la tolerancia mutua" (Declaración y programa de acción de Viena, 1993, acción 82).

Por último en cuanto a la Resolución 48/127 de la Asamblea de las Naciones Unidas, en ella, no sólo se reiteran los mismos aspectos, sino que se "exhorta a los órganos encargados de vigilar la observancia de los derechos humanos a que presten especial atención al cumplimiento por los Estados Miembros de su obligación internacional de promover la educación en la esfera de los derechos humanos" y, en particular, se "pide a la Comisión de Derechos Humanos que, en cooperación con los Estados Miembros, los órganos encargados de supervisar la aplicación de los tratados de derechos humanos, otros órganos apropiados y las organizaciones no gubernamentales competentes, examine propuestas para celebrar un decenio de las Naciones Unidas para la educación en la esfera de los derechos humanos" (Resolución 48/127 de la Asamblea General de las Naciones Unidas, 1993, 3).

Fruto precisamente de este último encargo fue la aparición, en 1994, de uno de los textos más importantes en la materia que nos ocupa: el informe del Secretario General sobre "Educación en la Esfera de los Derechos Humanos", presentado ante la Asamblea General el 18 de julio de ese año por delegación de la Comisión de Derechos Humanos, y que ofrece, quizá por vez primera, una definición de lo que debía entenderse por ésta. La “educación en materia de derechos humanos", señalaba el Informe, "puede definirse como las actividades de educación, capacitación, difusión e información dirigidas a crear una cultura universal de los derechos humanos mediante la transmisión de conocimientos y capacidades y la formación de posturas, todo ello con el propósito de: a) Fortalecer el 
respeto de los derechos humanos y las libertades fundamentales; b) Desarrollar plenamente la personalidad humana y el sentido de dignidad del ser humano; c) Promover la comprensión, la tolerancia, la igualdad entre los sexos y la amistad entre todas las naciones, los pueblos indígenas, los grupos raciales, étnicos y religiosos ; d) Permitir que todas las personas participen de forma eficaz en una sociedad libre"; y "e) Incrementar las actividades de las Naciones Unidas en la esfera del mantenimiento de la paz" (Preparación de un plan de acción para la celebración de un decenio de las Naciones Unida, 1994, 6-7).

Una educación, añadía el informe, que no sólo era ya claramente asumida por la ONU, muchos Estados y ONG, sino que contaba ya con algunas experiencias prácticas muy valiosas como las desarrolladas en años previos por el "Centro de Derecho Humanos", al impartir cursos sobre los mismos a determinados profesionales (jueces, militares, funcionarios,...) o al desarrollar actividades dirigidas a instruir al público en general (publicaciones, exposiciones, reuniones,...); una experiencia de la que se podían inferir, según el mismo texto, diversos recursos didácticos y evaluadores que podrían ser útiles para una educación en derechos humanos. Entre los primeros, el uso de "métodos pedagógicos creativos e interactivos" con el fin de "despertar el interés y lograr la participación activa" de los discentes, crear "grupos de trabajo", combinar "exposición y debate", estudiar "casos concretos", debatir "en grupos de expertos" y en "mesas redondas", presentar súbitamente "ideas inesperadas", simular e interpretar "distintos papeles", "visitas de campo", "labores prácticas" y, por supuesto "instrumentos de ayuda visual". Entre los segundos, “instrumentos de evaluación” previos, sincrónicos o posteriores a los cursos tales como "cuestionarios" y "reuniones de evaluación". Unos métodos que, en cualquier caso, siempre debían entenderse de forma flexible en su “diseño y aplicación” para conseguir un conocimiento profundo de los derechos humanos (Preparación de un plan de acción para la celebración de un decenio de las Naciones Unida, 1994, 19-21).

Se anexaba además al Informe un "Proyecto de plan de acción para el decenio de las Naciones Unidas para la educación en la esfera de los derechos humanos", en el cual, de forma más sistemática que en el propio informe, se trataba de lo que debían ser sus "principios rectores" (un enfoque amplio sobre todos los derechos humanos -civiles, culturales, económicos, políticos y sociales- entendidos de forma indivisible e 
interdependiente), sus “objetivos" (fundamentalmente evaluar el estado de la educación en derechos humanos y mejorarlo), protagonistas (esencialmente los gobiernos, las Organizaciones Internacionales y las ONG), grupos seleccionados (el máximo de público posible), y un "programa de ejecución" que lo convirtiera realmente en un Decenio fructífero con un seguimiento del Alto Comisionado con la asistencia del Centro de Derechos Humanos en cooperación con la UNESCO (Preparación de un plan de acción para la celebración de un decenio de las Naciones Unida, 1994, 29-50).

\subsection{El decenio de las Naciones Unidas para la educación en derechos humanos}

Dos nuevos pasos se dieron en este proceso de llevar a la práctica la educación en derechos humanos: uno de ellos fue, en octubre, la Declaración de los Ministros de Educación presentes en la $44^{\mathrm{a}}$ reunión de la Conferencia Internacional de Educación de la UNESCO, en la que se admitió la necesidad de mejorar "la educación relativa a los derechos humanos y las libertades fundamentales", especialmente entre niños y jóvenes, para evitar "las manifestaciones de violencia, racismo, xenofobia y nacionalismo agresivo, y las violaciones de los derechos humanos" (Declaración de los Ministros de Educación en la Conferencia Internacional de Educación de la UNESCO, 1994). La otra, la importantísima Resolución 49/184 de la Asamblea General de la ONU, en la que finalmente se proclamó un "Decenio de las Naciones Unidas para la educación en derechos humanos" que iría del 1 de enero de 1995 al de 2005, y que seguía el Plan de Acción trazado por el informe del Secretario General antes visto. Una Declaración que marcó un hito esencial en la historia de la educación en derechos humanos que de inmediato se tradujo en intentos de lograr su éxito.

El "Plan de Acción Integrado sobre la Educación para la Paz, los Derechos Humanos y la Democracia" adoptado en la $28^{\mathrm{a}}$ reunión de la Conferencia General de la UNESCO en 1995 es un buen ejemplo de ello, al desarrollar "políticas y líneas de acción" aún más concretas para facilitar la plasmación en la práctica de esta educación en derechos humanos, precisando el contenido que debía darse a esta educación, señalando que ha de favorecer "la adquisición de valores" (como la responsabilidad cívica, la solidaridad, la capacidad de resolver conflictos e incluso la creatividad), e insistiendo en que debía ser democrática (tanto en contenido como en el contexto institucional), intercultural, no 
discriminatoria, transversal al currículo (no sólo especializada), pero profunda y fundamentada (también histórica y jurídicamente).

Los "materiales y recursos pedagógicos", también eran objeto de atención en este plan, pidiéndose que fueran adecuados (desde "libros de texto" a los propios documentos internacionales), puestos al servicio de los agentes educativos (formados en derechos humanos y con una labor revalorizada) y con una potenciación de la cooperación internacional y los intercambios educativos tanto de discentes como de docentes. En cuanto a la didáctica, insistía en que "conviene fomentar la utilización de los métodos activos, las tareas en grupo, la discusión sobre cuestiones morales y la enseñanza personalizada". Y, en relación con la "Enseñanza Superior", instando a que las Universidades introdujeran en sus planes de estudio "conocimientos, valores y aptitudes referentes a la paz, los derechos humanos, la justicia, la práctica de la democracia, la ética profesional, el civismo y la responsabilidad social”, algo que además podría vincularse al fomento de la "Investigación y desarrollo" en estas materias (Plan de Acción Integrado sobre la Educación para la Paz, los Derechos Humanos y la Democracia en Actas de la Conferencia General de la UNESCO, 1995, 64-68).

La dificultad de llevar a la práctica todas estas indicaciones en el marco del Decenio para la educación en la esfera de los Derechos Humanos se puso, sin embargo, inmediatamente de manifiesto. Varias Resoluciones de la Asamblea General de la ONU (como la 50/177 de 22 de diciembre de 1995 o la 51/506 de 16 de octubre de 1996) dejaban entrever que el problema, como siempre, no estaba en las grandes declaraciones (incluso desarrolladas en planes más o menos concretos) sino que en llevarlas a la práctica. Conscientes de esta dificultad y para que la educación en derechos humanos se tradujera en cada uno de los Estados, una nueva Resolución de la Asamblea General de 20 de noviembre de 1997 (52/469/Add.1) acordó unas "directrices para la elaboración de planes nacionales de acción para la educación en la esfera de los derechos humanos" que fueran efectivos y realistas. Muy en resumen: que fueran planes que reconocieran la relevancia de la enseñanza de los derechos humanos (desde el reiterado fomento de la democracia al mantenimiento de la paz, pasando por nuevos objetivos como el desarrollo sostenible o la protección del medio ambiente), que tuvieran una visión de los mismos interdependiente, indivisible y universal (sin perjuicio de insistir en los derechos de colectivos más desprotegidos, como los de las mujeres), que establecieran una enseñanza 
profunda, con medios suficientes y en "entornos de aprendizaje libres de necesidades y temores", y que partieran del uso de "métodos didácticos" participativos, críticos, respetuosos e impartidos por docentes capacitados para ello.

El poco éxito de estas directrices fue, pese a todo, evidente $\mathrm{y}$, con ello, la propia virtualidad del Decenio. Un Informe del Alto Comisionado de las Naciones Unidas de 2004 en el que se indicaban a modo de balance final los "logros y fallos" que había tenido el Decenio lo deja meridianamente claro. Aunque en él se destacaban algunas actividades emprendidas (unos pocos planes de acción, algunas leyes de educación y programas aislados), lo cierto es que de forma mayoritaria el Decenio no había tenido el éxito deseado; algo que también se constata en el hecho de para la elaboración de este reporte sólo treinta países colaboraron dando información al respecto, y en el hecho de que, durante la vigencia del Decenio, algunas de las más importantes declaraciones internacionales aprobadas entonces, no trataron de conectarse claramente con él. Un ejemplo es la célebre "Declaración del Milenio" que, pese a incluir como uno de sus objetivos (el segundo) lograr para 2015 que "los niños y niñas de todo el mundo puedan terminar un ciclo completo de enseñanza primaria", no hacía referencia expresa a una educación en derechos humanos; una omisión que, aunque a posteriori, trató de solucionarse, responde a los defectos de los ODM de no captar otros compromisos existentes (HERFKENS, 2008) y de ser poco efectivos (GÓMEZ GIL, 2009, 58-59).

Por supuesto esto no quiere decir que el Decenio fuera un fracaso. Por vez primera se había logrado colocar en la palestra internacional como objetivo preferente, la educación en derechos humanos. Simplemente se demostraba que, como ocurre con otras grandes cuestiones planteadas por la ONU, era una materia compleja de llevar a la práctica. En este sentido, el Decenio debe entenderse más como un buen inicio que como un resultado en sí mismo. Por eso, el propio informe del Alto Comisionado antes indicado, planteaba una serie de "futuras iniciativas" con el objetivo precisamente de seguir ahondando en esta cuestión, desde la posibilidad de proclamar "un segundo Decenio para la educación en la esfera de los derechos humanos" a establecer "un fondo de contribuciones voluntarias" para el fomento de la misma (Informe del Alto Comisionado de las Naciones Unidas relativo a la decenio de las Naciones Unidas para la educación en la esfera de los derechos humanos, 2004, 9). 


\subsection{La educación en derechos humanos desde 2004 hasta el presente}

En respuesta al informe del Alto Comisionado anteriormente citado, el 10 de diciembre de 2004, la Asamblea General de las Naciones Unidas proclamó un Programa Mundial para la educación en Derechos Humanos. Dicho programa, se inició el 1 de enero de 2005 y en lugar de desarrollarse a lo largo de un decenio lo ha venido haciendo, hasta el presente, en sucesivas etapas, con el objeto de tener metas más concretas y viables. Coordinado por el ACNUDH y vinculado de forma expresa a la consecución de los ODM (tratando así de hacer sinergia con ellos), se han vivido ya cuatro fases en su desenvolvimiento: una primera, de 2005 a 2009, enfocada en la enseñanza primaria y secundaria; otra segunda, de 2010 a 2014, centrada esencialmente en el ámbito de los estudios superiores; una tercera, desarrollada entre 2015 y 2019, interesada en la educación en derechos humanos entre los periodistas y medios de comunicación; y una cuarta, en la que nos encontramos, a desarrollar entre 2020 y 2024, que se ocupa en esta educación entre los jóvenes.

De todas estas fases resulta de especial interés para este estudio, la segunda y la cuarta. La segunda, porque se centró especialmente en la educación en derechos humanos en el ámbito universitario. Respecto a la tercera, porque es la que actualmente está en vigor y, porque al enfocarse en la juventud, permite también una conexión con los estudios superiores.

El "Proyecto de plan de acción para la segunda etapa" se trata, en particular, de un texto interesante por varios motivos. En primer lugar, porque insiste en el carácter "fundamental" que tiene la educación en Derechos Humanos en el ámbito universitario (tanto en la docencia, como en la investigación). En segundo, porque al definirla, actualiza la definición de 1994 introduciendo en ella "la igualdad entre los géneros", "las minorías", "el desarrollo de una sociedad libre y democrática en la que impere el estado de derecho", "el desarrollo sostenible" y "la justicia social" (pp. 4-5). En tercero, porque insiste en el papel compartido que en su promoción tienen que tener tanto las universidades como los gobiernos y otros agentes (Docentes, discentes, ONGs, Sindicatos,...). En cuarto, porque ofrece diversas indicaciones acerca de cómo debe realizarse dicha docencia, insistiendo en todo caso en que "la introducción o el perfeccionamiento de la educación en derechos humanos en el sistema de enseñanza 
superior" debe suponer "la adopción de un enfoque holístico de la enseñanza y el aprendizaje” (p. 12). Y por último, porque, desde un punto de vista práctico, señala una serie de "aspectos" "necesarios para lograr una enseñanza y un aprendizaje de calidad en relación con los derechos humanos": la existencia de una programación adecuada de los mismos "en todas las disciplinas de la enseñanza superior" (mediante contenidos general o especiales, en todos los niveles de ella y con aportación suficiente de recursos); un enfoque multidisciplinar e interdisciplinar de los derechos humanos; la elaboración y uso de "materiales de enseñanza y formación equilibrados y pertinentes en relación con" ellos; y un método de enseñanza/aprendizaje siempre "coherente con los derechos humanos" (en un "entorno de aprendizaje" libre y respetuoso, de forma participativa, crítica y práctica) (p. 13).

El resultado de esta segunda etapa del Programa Mundial para la Educación en Derechos Humanos centrada en la enseñanza superior no alcanzó, sin embargo, tampoco los resultados deseables. El Informe que el ACNUDH presentó como balance del mismo en 2015 (gracias a la información remitida por un limitado número de veintiocho países) tradujo, desde luego, que en algunos Estados había auténticas "políticas de educación superior relativas a la educación en derechos humanos", pero que, ni mucho menos, esta era la generalidad. Se constataba con ello una vez más el salto entre la teoría (el consenso internacional de la importancia de una educación en derechos humanos, en este caso en la enseñanza superior) y una realidad muy diferente a la deseable, en la que la entrada efectiva de la educación en derechos humanos seguía siendo una quimera.

El cuarto periodo del "Programa Mundial para la educación en derechos humanos" en la que nos encontramos (2020-2024) vuelve a ser, por eso, un nuevo marco favorable para seguir avanzando en la educación en derechos humanos en la Universidad. Por un lado, porque, como se indicó, se centra "en los jóvenes, haciendo especial hincapié en la educación y formación sobre la igualdad, los derechos humanos y la no discriminación" (destinatarios y temáticas muy vinculadas a las Universidades); por otro, porque dicha educación "estará en consonancia con la Agenda 2030 para el Desarrollo Sostenible y, en particular, con la meta 4.7 de los Objetivos de Desarrollo Sostenible" de conseguir para 2030 "asegurar que todos los alumnos adquieran los conocimientos teóricos y prácticos necesarios para promover el desarrollo sostenible, entre otras cosas mediante la educación" (en) “derechos humanos". 
Ciertamente la educación en derechos humanos no es, de forma expresa, ninguno de los ODS (menos aún en el ámbito universitario), pero es evidente la sinergia que puede darse entre ellos (GIL PÉREZ, D. y VILCHEZ, A, 2017): los ODS, como antes los ODM, pueden desarrollarse mejor con una educación en Derechos Humanos; la Educación en Derechos Humanos, por su parte, puede afianzarse definitivamente si se aprovecha el interés que dichos objetivos generan entre los jóvenes. De este modo, a través de "uno de los grandes retos a los que se enfrentan las sociedades del siglo XXI” (LÓPEZ PARDO, 2015, 112), la Educación en Derechos Humanos puede demostrar su utilidad práctica en la mejora del mundo.

\section{La enseñanza en derechos humanos en la universidad española}

La enseñanza en derechos humanos en las Universidades españolas ha sido acusada de graves insuficiencias desde principios del siglo XXI, pese al compromiso teórico de España de desarrollarla tras apoyar todas las iniciativas internacionales vistas anteriormente.

En su informe "Educación en derechos humanos: asignatura suspensa" de febrero de 2003, la Sección española de Amnistía Internacional (AI), centrándose en el estado de la enseñanza en derechos humanos "en las escuelas de magisterio y facultades de pedagogía y ciencias de la educación" concluía que (tras la consulta de los planes de estudio y realizar varias encuestas a estudiantes y profesores de una veintena de ellas) la educación dada a los docentes en nuestro país era claramente insuficiente en esta materia: "la educación en derechos humanos no figura como asignatura en los planes de estudio", "más de la mitad del profesorado desconoce el contenido de las normas y guías de acción que" les afectan "en el ámbito de la educación en Derechos Humanos" y "el alumnado de Escuelas de Magisterio y Facultades de Pedagogía y Ciencias de la Educación no sale preparado en cuanto a conocimientos de Derechos Humanos" (Informe, 2003, 42).

La rotundidad del informe resulta llamativa. "La investigación (...) constata que la educación en derechos humanos no es tomada en serio por el Gobierno español ni, en buena parte, tampoco por las autoridades autonómicas", incumpliéndose así "con las recomendaciones de las Naciones Unidas en cuanto a los compromisos del Decenio de 
las Naciones Unidas para la educación en la esfera de los Derechos Humanos 1996/2004)" (Informe, 2003, 42).

Para mejorar esta situación, la Sección Española de AI hacía una serie de recomendaciones: en primer lugar, recomendaba tanto al Gobierno español como a los autonómicos poner en marcha de forma inmediata "las directrices del Decenio", elaborando "un Plan de Acción Nacional” o por Comunidades Autónomas de tipo ejecutivo sobre estas materias, así como establecer Comités que pudieran evaluar la situación, crear programas educativos en materia de DDHH y preparar "materiales didácticos y guías para los educadores". En segundo lugar, sugería "a las autoridades académicas" introducir en los "planes de estudios" "materias relacionadas con la educación en Derechos Humanos" y promover su virtualidad. Y, por último, instaba "al profesorado" a que "asumiera la responsabilidad de capacitar" a los "futuros educadores" "para que transmitan y promuevan la educación en derechos humanos en todas sus actividades y ámbitos" y que pasaba no sólo por presionar a las "autoridades académicas" para mejorar la situación, sino también para que se formasen o reciclasen "en conocimientos sobre derechos humanos y educación en derechos humanos" $\mathrm{y}$ promovieran "actividades relacionadas con la educación en derechos humanos entre el alumnado, especialmente en relación con discriminación de la mujer y de grupos vulnerables (inmigrantes, minorías sexuales, etc.)" (Informe, 2003, 44-45).

Que la situación de la educación en derechos humanos fuera tan insuficiente en la enseñanza superior dedicada precisamente a la investigación y formación de los educadores nos puede hacer intuir cómo era de mala la situación también en el resto de los estudios universitarios y no universitarios (NAYA GARMENDIA, 2005; RIBOTTA, $S, 2006)$. De este modo, pese a que de inmediato algunas normas jurídicas trataron de impulsarla de forma genérica o en otros ámbitos educativos (la Ley 27/2005 de 30 de noviembre de fomento de la educación y la cultura de la paz, o la Ley Orgánica 2/2006 de Educación que introdujo la asignatura de Educación para la ciudadanía), un nuevo informe de la Sección Española de AI de mayo de 2008 constató nuevamente este hecho.

En dicho informe, titulado precisamente "Las Universidades españolas, a la cola de Europa en formación obligatoria en Derechos Humanos", se insistía en denunciar el desinterés de la Universidad española por la educación en Derechos Humanos, ya no sólo 
en las Facultades de Educación (en las que la situación seguía siendo la misma que en 2003) sino en otros ámbitos de la Universidad española, en concreto “Derecho, Medicina, Trabajo Social y Periodismo" (Informe, 2008, 6). De este modo, aunque se constataba la existencia de unas pocas asignaturas relativas a los DDHH en Derecho, Trabajo Social o Periodismo, su número seguía siendo escaso y en muchos casos eran asignaturas optativas, siendo aún menor en las Facultades de Educación (donde su estudio se reduce a enfoques demasiado globales) y de Medicina (limitada a asignaturas de valores como "Bioética o Ética médica"). Desde luego, muy por debajo de lo que ocurría en otras Universidades Europeas, en las que no sólo había más asignaturas sobre derechos humanos sino donde, en general, era mayor su carácter obligatorio y además de carácter más específico y poco global (centrados sólo en "derechos humanos específicos") (Informe, 2008, 12).

Con ello volvía pues a constatarse el incumplimiento sistemático de los compromisos internacionales asumidos por España en materia de educación en Derechos Humanos. Como indicaba el Informe: "España, como Estado parte de los acuerdos internacionales de derechos humanos y del Programa Mundial para la Educación en Derechos Humanos, tiene la obligación de cumplir y hacer cumplir las indicaciones relativas a la educación en derechos humanos y la formación del personal docente. Sin embargo, Amnistía Internacional ha podido documentar cómo el Estado español incumple su responsabilidad con relación al Plan de Acción del Programa Mundial (2005-2009) en lo que se refiere a la formación en derechos humanos del personal docente y estudiantes universitarios" (Informe, 2008, 12).

Pese a ello el informe se mostraba optimista. Inmersa España en aquel momento en un proceso de adaptación al Espacio Europeo de Educación Superior surgido a raíz de la Declaración de Bolonia, que implicaba la sustitución de las antiguas licenciaturas por nuevos Grados para 2010/2011 (y en las que incluso el Real Decreto 1393/2007 sobre la ordenación de las enseñanzas universitarias indicaba -art. 3- que los planes de estudio universitarios debían "incluir contenidos relacionados con los derechos humanos"), la denuncia del Informe podía ser entendida como una oportunidad para corregir estos defectos al elaborarse los nuevos Grados. Por eso la Sección Española de AI volvía a instar ahora al Gobierno español y a los autonómicos para que promovieran la entrada de contenidos de derechos humanos en los programas educativos (de Grado y de Máster) 
como requisito para la verificación de los títulos (Informe, 2008,13). Y, especialmente, a las Autoridades universitarias para que hagan lo mismo, introduciendo "al menos una asignatura obligatoria de educación en derechos humanos en todas las titulaciones de Educación, enfocada a una correcta impartición en Educación Primaria de la asignatura de Educación para la Ciudadanía y los Derechos Humanos” (Informe, 2008,14). Y “una asignatura obligatoria de derechos humanos en los grados de Periodismo, Medicina, Trabajo Social y Derecho. Dicha materia debe estar orientada al conocimiento general de los acuerdos nacionales e internacionales sobre derechos humanos, haciendo especial hincapié en aquellos referidos directamente a su titulación” (Informe, 2008, 18).

Las expectativas de la Sección Española de AI, por desgracia, no se cumplieron de forma generalizada. Aunque el 12 de diciembre de 2008 se aprobó por Acuerdo del Consejo de ministros un Plan de Derechos Humanos "con vocación educativa" (p. 5) y se incluyeron medidas concretas sobre "formación en derechos humanos" a los "actores de la Justicia" (medidas 80-82) y a las Fuerzas y Cuerpos de Seguridad del Estado (medidas 103-105), las referencias concretas a la "enseñanza de los derechos humanos en los centros docentes" (medidas 117-125) se redujo en esencia a la educación preuniversitaria (en particular, la implementación de Educación para la Ciudadanía) y a la formación de sus docentes (insistiendo exclusivamente en la incorporación de una educación en derechos humanos en las Facultades de Educación y en el Máster habilitante de los docentes de Secundaria). De este modo, muchos Grados de los indicados en el Informe de AI (y otros de Ciencias Sociales y Jurídicas) se diseñaron sin incluir asignaturas obligatorias en derechos humanos, siendo lo más común incluir éstos como parte del contenido de otras asignaturas más amplias, o simplemente diluyéndolos en una mera referencia a su transversalidad $^{16}$. Incluso, como advertía hace sólo dos años BELLVER "hay una

\footnotetext{
${ }^{16}$ A modo de ejemplo, en la Universidad de Málaga, la Memoria de Verificación del Grado en Derecho no incluye ninguna asignatura dedicada exclusivamente a la enseñanza de los derechos humanos. De hecho, esta expresión, sólo aparece una vez como parte del contenido de una asignatura optativa ("Instituciones Jurídicas Históricas"), mientras la expresión "derechos fundamentales" aparece de forma muy genérica al tratar de los requisitos de acceso al título y en otras pocas asignaturas: en "Proceso penal y derechos fundamentales", "Derecho Procesal II" y "Derecho Constitucional Europeo y Comparado". En la Memoria de Verificación del Grado en Relaciones Laborales y Recursos Humanos no se habla expresamente de "derechos humanos" y la única referencia que se hace a los "derechos fundamentales" es para indicar que se vincularán al ámbito de las relaciones laborales como orientación general del título y como competencia a adquirir en él. Mientras en el Grado en Gestión y Administración Pública, la referencia se limita a hablar del respeto a los derechos fundamentales en el acceso a la carrera. Por supuesto, esto no quiere decir que no se hable luego de los derechos humanos en diversas asignaturas de estos Grados, simplemente confirma que no se consideran un objetivo prioritario e individualizado de estudio.
} 
mayoría de grados que no incluyen referencia alguna de los derechos humanos en sus planes de estudio" (BELLVER, 2019, 18). De este modo, se perdió una oportunidad esencial para haber conseguido consolidar la educación de derechos humanos en la Universidad Española.

Es cierto que la educación en derechos humanos tiene hoy en nuestro país más importancia que hace veinte años. Sin duda, se ha avanzado mucho en ella en todos los ámbitos y, también, en el universitario, pero no puede decirse que la situación sea la deseable. El esfuerzo del Gobierno por poner en marcha un II Plan de Derechos Humanos en diciembre de 2018, vinculado a la Agenda 2030, o el evidente aumento de la sensibilización que en los últimos tiempos se ha venido operando en la ciudadanía, especialmente en relación con los derechos del colectivo LGTBIQ, los de inmigrantes o los de las mujeres, no supone que exista una educación en derechos humanos realmente efectiva. Los linchamientos mediáticos a través de internet borrando toda presunción de inocencia, el aumento de juicios históricos revisionistas de carácter poco científico, la pérdida de las formas democráticas en algunos de nuestros políticos o la construcción de fórmulas teóricas excluyentes ("los derechos no se discuten”, algunas teorías de género radicales,...) son el mejor ejemplo de que queda aún mucho por hacer en materia de educación en derechos humanos, muy particularmente, en la Universidad.

\section{Didáctica de los derechos humanos y la perspectiva histórico-jurídica}

El aparente consenso que existe a favor de incluir en los sistemas educativos materias referentes a los Derechos Humanos es bastante más débil de lo que pudiera pensarse inicialmente. Como ha podido observarse en las páginas previas, una cosa es la defensa teórica de esta educación, para la cual existe un apoyo internacional mayoritario, y cosa distinta es luego llevarlo a la práctica. España es un claro paradigma de esta disparidad entre el querer y el hacer, entre apoyar grandes declaraciones internacionales y luego adoptar medidas realmente efectivas para llevarlas a la práctica.

\subsection{Los obstáculos para una educación en derechos humanos en el Sistema Educativo Español, en particular el universitario}


Como indiqué al principio de este trabajo tres son las grandes dificultades para implementar una educación en derechos humanos en todo sistema educativo, incluido el nuestro: la necesidad de adaptar la enseñanza en derechos humanos a diversos niveles educativos formales e informales; la disparidad de políticas educativas existentes para su enfoque (recordemos en España la polémica surgida, por ejemplo, en relación con la Educación para la Ciudadanía, acusada de "adoctrinadora” por los sectores sociales más conservadores) y la cuestión pedagógica, es decir, la forma concreta en que debe educarse en derechos humanos. Pero lo cierto es que dichas dificultades, no sólo aparecen profundamente imbricadas entre sí, sino que se complican y aumentan conforme tratamos de enfocarnos en una realidad más específica.

En concreto, las dificultades de llevar al aula universitaria el estudio de los derechos humanos en ciencias sociales y jurídicas, tanto en el marco ideal de una asignatura específica dedicada a ello, como especialmente en el de la transversalidad (cuando se introduce en una asignatura de otra materia), creo que pueden sintetizarse en las siguientes: en primer lugar, en el pudor del o de la docente a estar "adoctrinando" al alumnado (al tratar sobre valores); en segundo, a su sensación (fundada o infundada) de falta de preparación en derechos humanos (probablemente más común entre el profesorado de ciencias sociales que en el de las jurídicas); en tercero, a la falta de instrumentos didácticos para hacerlo; y por último, a la falta de impulso y coordinación real desde las autoridades académicas que vayan más allá de las grandes declaraciones programáticas.

En relación con el primero de los problemas no puede negarse que encierra cierta lógica inicial. La labor del o de la docente universitaria, puede afirmarse, es esencialmente transmitir a estudiantes adultos un conocimiento especializado del que es experto, pero no unos valores éticos o morales que, se supone, ya ha elegido o no el alumnado libremente. El temor a adoctrinar, a inculcar desde una posición de autoridad en el aula valores que pueden ser considerados propios de quien imparte las clases, es un escrúpulo completamente explicable, sobre todo entre quienes son más tolerantes y respetan la libertad del otro, pero equivocado: por un lado porque educar en derechos humanos nunca debe suponer imponer valores sino explicarlos y debatirlos, como más tarde se argumentará al entrar en su didáctica; por otro lado, porque sea cual sea la docencia y por objetiva que quiera hacerse los valores de quien da clase siempre se traducirán de algún 
modo en el aula. Por supuesto, el o la docente que tenga estos escrúpulos, debe además tener la plena seguridad de que estos reparos adoctrinadores que se plantea, no los tienen aquellos que se muestran en contra de los valores que dichos derechos representan.

La falta de preparación del docente para impartir derechos humanos es otro escollo importante. Aunque reiteradamente se ha insistido desde instancias internacionales en que es labor de las autoridades políticas y académicas facilitar esta formación, lo cierto es que hoy sigue siendo un tema esencialmente dejado a la voluntad del docente. Es él, quien debe buscar su fuente de formación, y aunque eso es hoy relativamente sencillo hacerlo si hay interés por su parte (una bibliografía abundante, cursos diversos y gratuitos en internet, portales de organismos internacionales y ONG) lo cierto es que favorece un conocimiento a veces insuficiente y fraccionado. Sin duda deberían ser las Universidades las que ofrecieran esta formación de forma reglada, constante y reactualizada, y si no de forma obligatoria, al menos con la misma insistencia con la que ya piden su formación en otros ámbitos (por ejemplo, idiomas) o al tiempo invertido en otras actividades burocráticas. Quizá la presencia de incentivos reales en la tan poco valorada actividad docente, podría ayudar al respecto.

\subsection{La didáctica de los derechos humanos en el aula: especial referencia a la historia como recurso educativo.}

En la didáctica de una Educación de los Derechos Humanos son esenciales varias cosas: entenderlos y enseñarlos como una realidad histórica sin desvincularlos del presente; usar una pluralidad de medios para enseñarlos que favorezcan el dialogo y la crítica; y, además, hacerlo en un contexto respetuoso con los derechos humanos.

El primero de estos aspectos, el del carácter histórico de los derechos humanos, no suele destacarse en los textos internacionales, pero sí ha sido desarrollado por multitud de autores (PECES-BARBA MARTÍNEZ, 2001; CARIDE GÓMEZ, 2007; etc.). Destacar la naturaleza histórica de los derechos humanos supone evitar el dogmatismo de presentarlos como lo que no son (realidades sin pasado, innatas e indiscutibles), a favor de mostrarlos como lo que son en realidad (un producto cultural, cambiante, discutible y frágil). Es muy común entre discentes e incluso docentes, entender justo lo contrario. Parte de la culpa de esta visión ahistórica la tienen las propias declaraciones de derechos 
tradicionales (en particular la Francesa de 1789 y su fundamento metafísico amparado en un "Ser Supremo"), pero también la tienen la comodidad que resulta de explicar algo indiscutido (como si fuera una fórmula científica o un catecismo que no admite réplica), así como la falta de conocimiento del pasado y la existencia de creencias erróneas pero muy enraizadas, como la de entender que el liberalismo es una corriente superada y desvinculada de la creación de los derechos humanos, creer que éstos pueden ser compatibles con todas las culturas o que toda democracia los reconoce siempre.

Los derechos humanos son, como ya señalé al principio de estas páginas, un producto cultural, un producto histórico con una partida de nacimiento muy concreta: el nacimiento y desarrollo del primer liberalismo. Surge ciertamente también por otra serie de circunstancias interconectadas como el desarrollo de la empatía en el siglo XVIII, pero no deja de ser un producto cultural de la civilización occidental y en concreto del liberalismo. Por eso es tan importante comprender realmente lo que significa este término y tan esencial separarnos de aquellas posturas que lo reducen sólo a la libertad del mercado y al capitalismo, porque de hacerlo así, corremos el peligro de dejar huérfanos a los derechos humanos de la filosofía que los planteó inicialmente y los plasmó en la práctica jurídica (MANENT, 1995; LECUONA PRATS, 2012).

Entendiendo los derechos humanos como realidades históricas, se pierde sin duda su principio de autoridad irracional (son discutibles) y por tanto se imposibilita su docencia unidireccional, centrada sólo en el docente, pero se descubre su auténtica naturaleza como logro cultural, como meta humana, como realidad compleja que exige contextos determinados (sistemas liberales democráticos). Se cierra la puerta de un estudio meramente dogmático de estos, pero se abre la posibilidad de enseñarlos a través del diálogo, la razón e incluso la controversia (MAGENDZO-KOLSTREIN y TOLEDOJOFRÉ, 2015): un asunto en el que todos tenemos algo que decir y en cuya construcción podemos definitivamente colaborar. De ahí la importancia de aproximarse a ellos también de forma directa, sin intermediarios: consultar las propias fuentes, las normas que los establecen y comprender que sólo se lograron después de una ardua lucha. Pero también la conclusión de que pese a ser productos históricos son también realidades vivas. Por eso es tan importante en su docencia traerlos al presente, ver lo que aún queda por hacer con ellos y los retos que hoy implican, en definitiva, traerlos a la práctica y a la realidad, a 
temas que interesen a los estudiantes, como los ODS (cambio climático, ecología, pobreza, etc.).

Se trata en definitiva de que el estudiante haga propia la dinámica del derecho humano, que lo vea como un elemento positivo y no como una imposición. Que puedan aplicar lo aprendido a sus propias vidas. Aquí creo que es muy útil leer informes internacionales como los de AI y, también, acudir a webs que nos enseñen a ser más conscientes y mejorar las cosas desde nuestra realidad individual, como la curiosa "Guía de los vagos para salvar el mundo" de la ONU relacionada con los ODS.

Queda claro, por tanto, tal y como se ha venido insistiendo durante años por la comunidad internacional, que la didáctica en el aula de los derechos humanos debe consistir en métodos participativos que fomenten la curiosidad, el diálogo y la crítica. También la emoción, pues como valores, están completamente vinculados a la empatía, a ponernos en lugar del otro. Por eso, el listado de los instrumentos didácticos para la educación de los derechos humanos sólo puede ser indicativo. Por una parte, porque pueden y deben combinarse entre sí. Por otro lado, porque pueden y deben adaptarse a cada materia y al carácter específico o transversal de los derechos humanos en cada asignatura concreta.

El uso de materiales de lectura adecuados es desde luego esencial si queremos llegar a un conocimiento superior, universitario de los derechos humanos. Estos materiales pueden ser textos especialmente dirigidos a la enseñanza de los derechos humanos en general o vinculados a una materia concreta; estudios de historia, tanto de los derechos, como de los movimientos de lucha que han logrado conseguirlos o biografías de sus líderes; la lectura de las propias normas jurídicas que los reconocen; o el estudio de informes internacionales o nacionales sobre el estado de los derechos (de la ONU y ONG). Incluso puede incluir otras fórmulas: artículos periodísticos, ensayos, novelas, obras audiovisuales (cine, documentales,...) u otras obras de creación artística que, de algún modo, puedan hacer reflexionar o aproximarse a los derechos humanos desde un punto de vista emocional.

El uso del debate también es esencial al menos en el ámbito universitario. Debatir acerca de la evolución en cascada de los derechos humanos o, especialmente, sobre los conflictos que puede haber entre ellos, resulta enormemente formativo e incentivador para los 
estudiantes. Al fin y al cabo, los derechos humanos son valores y éstos deben ser convincentes y no impuestos. Este método puede, ciertamente, generar algún conflicto en el aula y esto es algo que con toda lógica el docente ha de prever. ¿Qué ocurre cuando el diálogo no es convincente? ¿qué ocurre cuando al final se niega el derecho humano? Pues que también ha de enseñarse lo evidente: que los derechos humanos son también "derecho", es decir, un instrumento coercitivo.

Las conferencias, visitas de ONG al aula, trabajos en equipo o individuales centrados en temas actuales y de interés para los estudiantes como pueden ser los ODS, son recursos adecuados si la aproximación a los derechos humanos también lo es. Lo esencial será siempre que se usen en entornos educativos que no resulten contradictorios con lo que se enseña, es decir, en marcos adecuados de libertad y respeto, tanto de la Universidad, como, en concreto, del profesor. Es absurdo explicar derechos humanos si luego la institución universitaria o el docente es injusto y arbitrario con sus estudiantes. Así, si se comparte la idea de que "la educación en derechos humanos tiene un enorme potencial en la creación de ciudadanos activos" (CARIDE GÓMEZ, 2007, 326, siguiendo a LÓPEZ LÓPEZ, 2005, 161) deben ir quitándose aquellas realidades universitarias en que el estudiante se siente ninguneado o no se favorece la reflexión y su propia voz.

Las políticas educativas de la propia Universidad y por supuesto las del Gobierno tanto regional como nacional son por ello esenciales más allá del compromiso del docente, pues "educar en los derechos humanos y para los derechos humanos trasciende las fronteras del quehacer pedagógico para inscribir sus realizaciones en el discurso y las prácticas políticas" (CARIDE GÓMEZ, 2007, 326). De este modo no sólo la universidad debe ser un marco adecuado, sino que deberían potenciar los derechos humanos velando por su completa inclusión en los currículos (no sólo en los Másteres de formación del profesorado de secundaria) (BELLVER, 2019, 21), controlando su adecuada impartición dentro del aula, coordinando las actividades que ya se producen y favoreciendo la formación de los docentes al respecto.

Y es aquí sinceramente donde creo que más queda por hacer y donde más peligros puede haber de involución en el futuro. Educar en derechos humanos no es sólo hacer grandes declaraciones institucionales, "presupone comprometer la educación con un proceso continuo y permanente al que se asocian valores e ideales que reivindican una profunda 
transformación de la sociedad" con la "aspiración a una convivencia libre, equitativa, solidaria, justa, democrática y pacífica" (CARIDE GÓMEZ, 2007, 326). Y este compromiso, es evidente, está lejos de ser asumido por todos. Al final, si los derechos humanos no se coordinan dentro de la Universidad, no se potencian como algo realmente vertebrado, podemos incurrir en lo que para Lucio era otra de las "grandes desviaciones que se pueden presentar en el quehacer educativo", la de hacer una "didáctica sin pedagogía" (LUCIO, 1989, 43), es decir, enseñar derechos humanos sin una perspectiva pedagógica que asuma que la misma es una pieza específica de un proyecto más amplio: el de formar ciudadanos libres y empáticos, comprometidos con los derechos humanos y la democracia.

\section{Bibliografía}

ALTO COMISIONADO DE LAS NACIONES UNIDAS (2004). Informe relativo al Decenio de las Naciones Unidas para la educación en la esfera de los derechos humanos. 25 de febrero.

https://documents-ddsny.un.org/doc/UNDOC/GEN/G04/112/36/PDF/G0411236.pdf?OpenElement

ALTO COMISIONADO DE LAS NACIONES UNIDAS (2010). Proyecto de plan de acción para la segunda etapa del Programa Mundial de Acción para la Educación en Derechos Humanos. https://documents-ddsny.un.org/doc/UNDOC/GEN/G10/151/51/PDF/G1015151.pdf?OpenElement

ALTO COMISIONADO DE LAS NACIONES UNIDAS (2015). Informe sobre la evaluación de la aplicación de la segunda etapa del Programa Mundial para la Educación en Derechos Humanos. 16 de julio. https://documents-ddsny.un.org/doc/UNDOC/GEN/G15/158/96/pdf/G1515896.pdf?OpenElement

ASAMBLEA GENERAL DE LAS NACIONES UNIDAS (1993)._Resolución 48/127. 29 de diciembre. file:///C:/Users/USUARIO/Downloads/A_RES_48_127-ES\%20(1).pdf

ASAMBLEA GENERAL DE LAS NACIONES UNIDAS (1995). Resolución 49/184. 6 de marzo, por la que se proclama un "Decenio de las Naciones Unidas para la educación en la esfera de los derechos humanos". https://undocs.org/es/A/RES/49/184 
ASAMBLEA GENERAL DE LAS NACIONES UNIDAS (1995). Resolución_50/177. 22 de diciembre de 1995. https://undocs.org/pdf?symbol=es/A/RES/50/177

ASAMBLEA GENERAL DE LAS NACIONES UNIDAS (1996). Resolución_51/506. 16 de octubre. file:///C:/Users/USUARIO/Downloads/A_51_506-ES.pdf

ASAMBLEA GENERAL DE LAS NACIONES UNIDAS (1997). Resolución 52/469/Add.1. 20 de noviembre. https://documents-ddsny.un.org/doc/UNDOC/GEN/N97/284/14/PDF/N9728414.pdf?OpenElement

ASAMBLEA GENERAL DE LAS NACIONES UNIDAS (2004). Resolución 59/113. Proclamación de un Programa Mundial para la Educación en Derechos Humanos. 10 de diciembre. https://documents-ddsny.un.org/doc/UNDOC/GEN/N04/483/07/PDF/N0448307.pdf?OpenElement

ASAMBLEA GENERAL DE LAS NACIONES UNIDAS (2000). Declaración del Milenio. 8 de septiembre. https://www.un.org/spanish/milenio/ares552s.htm

ASAMBLEA GENERAL DE LAS NACIONES UNIDAS (2015) Agenda 2030 para el Desarrollo Sostenible.25 de septiembre de 2015. https://unctad.org/system/files/officialdocument/ares70d1_es.pdf

BELLVER CAPELLA, V. (2019) Educar en Derechos Humanos: orientaciones del Derecho internacional e implementación en la educación superior. Revista de educación y derecho. $\mathrm{N}^{\mathrm{o}}$ 20, 2, 1-25.

CARIDE GÓMEZ, J. A. (2007). Derechos Humanos y Políticas Educativas. Bordón. Revista de Pedagogía, 59 (2-3) 313-334.

CONFERENCIA INTERNACIONAL DE DERECHOS HUMANOS (1968). Teherán, 22- 13 Mayo. https://legal.un.org/avl/pdf/ha/fatchr/Final_Act_of_TehranConf.pdf 
CONFERENCIA MUNDIAL SOBRE EDUCACIÓN PARA TODOS (1990) Jomtién, Tailandia, 5-9 de marzo. https://unesdoc.unesco.org/ark:/48223/pf0000127583_spa

CONFERENCIA MUNDIAL DE DERECHOS HUMANOS (1993). Declaración y programa de acción de Viena. 20 años trabajando por tus derechos. https://www.ohchr.org/Documents/Events/OHCHR20/VDPA_booklet_Spanish.pdf

COMISIÓN DE DERECHOS HUMANOS DE LA ONU (1977). Resolución 3/1977. Further promotion and encouragement of human rights and fundamental freedoms, including the question of the programme and methods of work of the Commission. Commission on Human Rights report on the thirty-third session (7 February-11 March 1977), Economic and Social Council, Official Records: Sixty-second session,

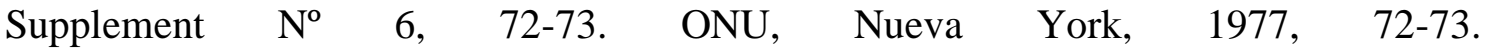
https://undocs.org/pdf?symbol=en/E/5927(SUPP)

COMISIÓN DE DERECHOS HUMANOS DE LA ONU (1993)._Resolución 56/1993 de la Comisión de Derechos Humanos, sobre "Educación y derechos humanos" que se encuentra entre las pág. 193 -195 en la Comisión de Derechos Humanos, Informe sobre el $49^{\circ}$ Periodo de sesiones $\left(1^{\circ}\right.$ de febrero a 12 de marzo de 1993). Consejo Económico y Social, 1993, Suplemento $\mathrm{n}^{\mathrm{o}}$ 3, pp. 193-195.ONU, Nueva York, 1993. https://digitallibrary.un.org/record/168468? $\ln =e n$

CONSEJO DE DERECHOS HUMANOS (2018). Resolución de 27 de septiembre referente al cuarto periodo del Programa Mundial para la educación en derechos humanos (2020-2024).

https://documents-ddsny.un.org/doc/UNDOC/GEN/G18/293/78/PDF/G1829378.pdf?OpenElement

GIL PÉREZ, D. y VILCHES, A. (2017). Educación para la sostenibilidad y educación en derechos humanos: dos campos que deben vincularse. Teoría de La Educación. Revista Interuniversitaria, 29(1), 79-100. doi:10.14201/teoredu29179100

GOBIERNO DE ESPAÑA (2008). Plan de Derechos Humanos. Aprobado por el Consejo de ministros. Gobierno de España. 12 de diciembre. https://www.idhc.org/arxius/incidencia/1424254612-PlanDH.pdf 
GÓMEZ GIL, C. (2009) Claves para una mejor interpretación de los objetivos de desarrollo del milenio a la luz de la crisis económica global, Revista Española de Relaciones Internacionales. $\mathrm{N}^{\mathrm{o}} 1$. 48-85. https://dialnet.unirioja.es/servlet/articulo?codigo $=4844040$

HERFKENS, E. (2008). “La Campaña del Milenio: Resultados positivos y dificultades en la movilización de apoyo en favor de los ODM", Partnership for Development, UN Chronicle, $\mathrm{N}^{\mathrm{o}} 1$, Vol. XLV. https://www.un.org/es/chronicle/article/la-campana-del$\underline{\text { milenio-resultados-positivos-y-dificultades-en-la-movilizacion-de-apoyo-en-favor-de }}$

HUNT, L. (2009). La invención de los derechos humanos. Barcelona: Tusquets.

LECUONA PRATS, E. (2012) La naturaleza histórica de los Derechos Humanos: entre la invención y el normativismo jurídico, en "Concepto y Fundamentos críticos", Cultura de paz, conflictos, educación y derechos humanos, coordinado por $\mathrm{M}^{\mathrm{a}}$. Teresa Castilla Mesa, Victor M. Martín. Ed. GEU, 154-158.

LÓPEZ LÓPEZ, P. (2005). Educación en derechos humanos: suspenso. En L. M. NAYA (coord.), La educación y los derechos humanos. Donostia: Erein: 155-173.

LÓPEZ PARDO, I (2015). Sobre el desarrollo sostenibles y la sostenibilidad: conceptualización y crítica. Barataria. Revista Castellano-Manchega de Ciencias Sociales, (20),111-128. https://www.redalyc.org/articulo.oa?id=322142550007.

LUCIO, R. (1989). Educación y Pedagogía, Enseñanza y Didáctica: diferencias y relaciones. Revista de la Universidad de La Salle, (17), 35-46.

MAGENDZO-KOLSTEIN, A. y TOLEDO-JOFRÉ, M. I. (2015) Educación en derechos humanos: Estrategia pedagógica-didáctica centrada en la controversia. Revista Electrónica Educare (Educare Electronic Journal) Vol. 19 (3), septiembre-diciembre, 116. doi: http://dx.doi.org/10.15359/ree.19-3 
MANENT, P. (1995). An intelectual history of Liberalism. Princeton, New Jersey: Princeton University Press

NAYA GARMENDIA, L. M. (coord..) (2005). La educación y los derechos humanos. Donostia: Erein

ONU (2020). Guía de los vagos para salvar el mundo. https://www.un.org/sustainabledevelopment/takeaction/

PECES-BARBA MARTÍNEZ, G. (coord..) (2001). Textos básicos de derechos humanos. Con estudios generales y especiales y comentarios a cada texto nacional e internacional. Madrid: Aranzadi

RIBOTTA, S (ed.) (2006). Educación en derechos humanos. La asignatura pendiente. Madrid: Dykinson.

RUIZ, G. R. (2020). Do Direito à Educação e seu desenvolvimento conceitual do ponto de vista histórico. Cadernos De História Da Educação, 19(2), 544-560. https://doi.org/10.14393/che-v19n2-2020-16

UNESCO (1974). Recomendación sobre la Educación para la Comprensión, la Cooperación y la Paz Internacionales y la Educación relativa a los Derechos Humanos y las Libertades Fundamentales, Actas de la Conferencia General, $18^{a}$ reunión París, 17 de octubre-23 de noviembre de 1974, Volumen 1, 153-161. UNESCO, 1975. https://unesdoc.unesco.org/ark:/48223/pf0000114040_spa?posInSet=7\&queryId=94227 4e7-7bee-4e53-98db-5f3990cd0b68

UNESCO (1978). Decisiones aprobadas por el Consejo Ejecutivo de la Unesco en su $105^{\mathrm{a}}$ reunión (París, 25 de septiembre-28 de noviembre). https://unesdoc.unesco.org/ark:/48223/pf0000036656_spa?posInSet=1\&queryId=2f517 a59-9120-4667-a325-c9e64bda044a

UNESCO (1993)._Plan Mundial de Acción para la educación en pro de los derechos humanos y la democracia. Congreso Internacional sobre la Enseñanza de los Derechos 
Humanos y la Democracia.

$8-11$

de

marzo.

https://www.ohchr.org/EN/Issues/Education/Training/Compilation/Pages/10.WorldPlan ofActiononEducationforHumanRightsandDemocracy(TheMontrealDeclaration)(1993).a $\underline{\operatorname{spx}}$

UNESCO (1994)._Declaración de los Ministros de Educación presentes en la 44ª reunión de la Conferencia Internacional de Educación. 3-8 octubre. http://www.ibe.unesco.org/sites/default/files/44th_DECLARATION_sp.pdf

UNESCO (1995). Plan de Acción Integrado sobre la Educación para la Paz, los Derechos Humanos y la Democracia. Actas de la Conferencia General de la UNESCO, $28^{\text {a }}$ reunión, París, 25 de octubre-16 de noviembre de 1995, Volumen 1, Resoluciones, pp. 64-68, 1996.

https://www.observatorioreligion.es/upload/74/81/Declaracion_Principios_Tolerancia__1995_UNESCO.pdf

SECCIÓN ESPAÑOLA DE AI (2003)._Informe de la Sección española de AI. Educación en derechos humanos: asignatura suspensa. Febrero. https://doc.es.amnesty.org/

SECCIÓN ESPAÑOLA DE AI (2008)._Las Universidades españolas, a la cola de Europa en formación obligatoria en Derechos Humanos. Mayo. https://doc.es.amnesty.org/

SECRETARIO GENERAL DE LAS NACIONES UNIDAS._Preparación de un plan de acción para la celebración de un decenio de las Naciones Unidas para la educación en la esfera de los derechos humanos. Informe, Asamblea General, Consejo Económico y $\begin{array}{llll}\text { Social, } & \text { ONU, } & \text { A/49/261/Add.1, } & \text { E/1994/110/Add.1, }\end{array}$ https://undocs.org/es/E/1994/110/Add.1 